\title{
Molecular mechanism of DNA topoisomerase I-dependent rDNA silencing: Sir2p recruitment at ribosomal genes.
}

\author{
AUTHORS \\ Anna D'Alfonso ${ }^{(1)}$, Francesca Di Felice ${ }^{(1)}$, Valentina Carlini ${ }^{(1)}$, Christine M Wright ${ }^{(2)}$, \\ Marla I Hertz ${ }^{(2)}$, Mary-Ann Bjornsti ${ }^{(2) \star}$ and Giorgio Camilloni, ${ }^{(1,3)^{\star}}$

\section{AFFILIATIONS} \\ (1) Dipartimento di Biologia e Biotecnologie, Università di Roma Sapienza, Roma, \\ 00185, Italy \\ (2) Department of Pharmacology and Toxicology, University of Birmingham at Alabama, \\ Birmingham AL, 35294, USA \\ (3) Istituto Pasteur, Fondazione Cenci Bolognetti, Università di Roma Sapienza, Roma, \\ 00185, Italy
}

Anna D'Alfonso, dalfonsoanna@yahoo.it

Francesca Di Felice, francesca.difelice@uniroma1.it

Valentina Carlini, vale611@hotmail.it Christine M Wright, cwright7@uab.edu Marla I Hertz, marla.hertz@gmail.com Mary-Ann Bjornsti, bjornsti@uab.edu Giorgio Camilloni, giorgio.camilloni@umiroma1.it

* To whom correspondence should be addressed

Mary-Ann Bjornsti Tel: +1 2059344579; Email: bjornsti@uab.edu

Giorgio Camilloni. Tel: +39 0649912808. Fax: +39 0649912500. Email: giorgio.camilloni@umiroma1.it 


\begin{abstract}
Saccharomyces cerevisiae sir2 $\Delta$ or top $1 \Delta$ mutants exhibit similar phenotypes involving ribosomal DNA, including; i) loss of transcriptional silencing, resulting in noncoding (nc)RNA hyper-production from cryptic RNA Polymerase II promoters; ii) alterations in recombination; and iii) a general increase in histone acetylation. Given the distinct enzymatic activities of Sir2 and Top1 proteins, a histone deacetylase and a DNA topoisomerase, respectively, we investigated whether genetic and/or physical interactions between the two proteins could explain the shared rDNA phenotypes. We employed an approach of complementing top1 $\Delta$ cells with yeast, human, truncated and chimeric yeast/human TOP1 constructs, and assessing the extent of ncRNA silencing and histone H4K16 deacetylation. Our findings demonstrate that residues 115-125 within the yeast Top1p N-terminal domain are required for complementation of the top1 1 rDNA phenotypes. In chromatin immunoprecipitation (ChIP) and coimmunoprecipitation experiments, we further demonstrate the physical interaction between Top1p and Sir2p. Our genetic and biochemical studies support a model whereby Top1p recruits Sir2p to the rDNA, and clarifies a structural role of DNA topoisomerase I in the epigenetic regulation of rDNA, independent of its known catalytic activity.
\end{abstract}

\title{
Keywords: DNA topoisomerase I; Sir2p; Transcriptional silencing; histone acetylation; rDNA.
}




\section{INTRODUCTION}

In the budding yeast Saccharomyces cerevisiae, the ribosomal RNA genes

(rDNA) are arrayed in tandem repeats of 150-200 units on chromosome XII (1). Each repeated unit is interrupted by an Intergenic Spacer Region (IGS), which contains: i) a replication origin (ARS, for autonomous replicating sequence); ii) a promoter of the $35 \mathrm{~S}$ RNA gene that is transcribed by RNA polymerase I (Pol I), iii) the 5S RNA gene transcribed by RNA polymerase III (Pol III); and iv) two cryptic non-coding RNA promoters (E-pro and C-pro), which are transcribed at low levels by RNA polymerase II (Pol II). In addition, each unit includes a replication fork barrier (RFB). Binding of the Fob1 protein to RFB sites selectively stalls the movement of replication forks in only direction (2). This asymmetric stalling of only one of the bi-directional replication forks at Fob1p-bound RFB sites avoids the adverse effects that would otherwise result from head-on collisions between the advancing replication machinery and transcription bubbles. At RFBs, recombination events also occur that maintain the homogeneity of the rDNA units. Dysregulation of these events leads to change in unit copy number and/or the formation of extrachromosomal rDNA circles (ERCs) (3).

The locus of tandem rDNA repeats is a highly active region of the genome at which concurrent DNA replication, transcription and recombination occurs (4). Consequently, strict control of polynucleotide polymerization and recombination are necessary to maintain rDNA stability. In this regard, the epigenetic state of rDNA chromatin, in particular the acetylation of histone $\mathrm{H} 4$ at residue Lys16 (H4K16) (5), has been demonstrated to be crucial for the coordination of these enzymatic functions.

Sir2p is one of four Silent Information Regulator (SIR) genes in yeast that function in transcription silencing. However, Sir2p uniquely posseses NAD+-dependent 
histone deacetylase activity and is the only $S I R$ gene product to function in the nucleolus. Yeast strains deleted for the SIR2 gene (sir2A), exhibit several phenotypes involving rDNA gene dysregulation, such as the loss of transcriptional silencing $(6,7)$, which results in ncRNA hyper-production from the cryptic Pol II promoters, E-pro and Cpro (8), and alterations in recombination (9), as evidenced by changes in rDNA unit copy number or elevated production of ERCs (10). Furthermore, sir2 $\Delta$ mutants exhibit a general increase in histone acetylation of rDNA chromatin, particularly at H4K12, H4K16 and $\mathrm{H} 3 \mathrm{~K} 9$ residues (11). Indeed, the rDNA defects observed in sir2 $\triangle$ mutants are consistent with the loss of Sir2p histone deacetylase activity $(12 ; 13 ; 14 ; 15)$.

Deletion of the TOP1 gene, which encodes DNA topoisomerase 1 (Top1p), also impacts rDNA, and the features of these top $1 \Delta$ phenotypes almost completely overlap those observed in sir2 $\Delta$ mutants. Notably, rDNA repeat hyper-recombination, loss of rDNA transcriptional silencing and the hyperacetylation of histone residues have all been reported in top1 $\Delta$ mutants $(16 ; 17 ; 18 ; 19 ; 20)$. The gene products of $S / R 2$ and TOP1 represent distinct enzymatic functions; the NAD+-dependent deacetylase activity of Sir2p and the alterations in the linkage or intertwining of DNA strands catalyzed by DNA topoisomerase I (Top1p), with no obvious homology shared between the two enzymes. Thus, it is not clear why the altered rDNA phenotypes exhibited by top $1 \Delta$ cells are so similar to those observed in sir2 $\Delta$ mutants.

In this work, we sought to determine how the loss of Top1p (in top1 $\Delta$ strains) causes alterations in phenotypes involving rDNA. In particular, we focused our attention on structural features of Top1p that impact epigenetic aspects of transcriptional silencing of ncRNA from cryptic E-pro and C-pro promoters and acetylation of histone H4K16 $(21 ; 5)$, using a strategy of complementing top $1 \Delta$ strains with a panel of TOP1 
variant genes, including a series of human/yeast $\mathrm{N}$-terminal top1 chimeras (22). Our results indicate a physical interaction of Top1p with Sir2p, and suggest that select residues in the $\mathrm{N}$-terminal domain of Top1p functions in the recruitment of Sir2p to the rDNA. This property of DNA topoisomerase I was independent of enzyme catalysis and illustrates a structural role for Top1p in the epigenetic regulation of rDNA.

\section{RESULTS}

Top1p functions in maintaining the extent of H4K16 acetylation and ncRNA silencing at the rDNA locus.

The loss of transcriptional silencing of rDNA repeats, and the increase in histone acetylation at the same locus, characterize both sir2 $\Delta$ and top $1 \Delta$ mutants in $S$. cerevisiae $(6 ; 7)$. The histone H4K16 residue is the main target of the Sir2p NAD+dependent deacetylase. In sir2 $\Delta$ mutants, hyperacetylation of this residue has been demonstrated to induce a loss of transcriptional silencing, and leads to genome instability at rDNA (5). While these molecular features are consistent with the loss of the deacetylating activity of Sir2p, the underlying mechanisms involving Top1p in these phenotypes are not obvious. To elucidate the role of Top1p in regulating transcriptional silencing and histone acetylation at rDNA, we first assessed the extent of H4K16 acetylation and quantified ncRNA production in a top1 $\Delta$ strain, which lacks Top1p.

The relative positions of the $35 \mathrm{~S}$ gene and the functional elements of the intergenic spacer region (IGS) of the $9.1 \mathrm{~Kb}$ rDNA unit are diagrammed in Fig. 1. Five distinct DNA tracts in the IGS of the rDNA, encompassing the RFB, the cryptic E-pro promoter, the $5 \mathrm{~S}$ promoter, an ARS, and the cryptic C-pro promoter (referred to as $1-5$, respectively) were analyzed by chromatin immunoprecipitation (ChIP) with an anti- 
H4K16ac antibody (Fig. 2A), in order to measure the extent of histone H4K16 acetylation. Relative to isogenic wild-type TOP1 cells, top $1 \triangle$ cells exhibit increased levels of H4K16ac throughout the IGS sequences analyzed (see dotted line in Fig. 2A). These results are consistent with those previously reported, which indicate an increase in histone acetylation at rDNA in a top1 $\Delta$ strain (19).

Previous observations demonstrate that rDNA histone acetylation levels correlate with increased ncRNA production by RNA polymerase II transcription from the normally cryptic E- and C- pro promoters $(8 ; 5)$. To further characterize the functional involvement of Top1p in ncRNA silencing, we measured the three ncRNAs from the IGS (detailed in Fig. 1). Total RNA from isogenic top1 $\triangle$ or wild-type TOP1 strains were subjected to semi-quantitative RT-PCR, normalized to UBC6 mRNA expression (23), and reported in the histogram of Fig. 2B. A significant $(p<0.05)$ increase in the production of the ncRNAs IGS1F and IGS1R from the E-pro promoter was evident in top1 $\Delta$ compared to wild-type cells (dotted line in Fig. 2B). However, in contrast, no significant differences in IGS2 ncRNA levels were detected between wild-type and top $1 \Delta$ cells. Taken together, the data reported in Fig. 2 indicate that $\mathrm{H} 4 \mathrm{~K} 16$ acetylation within the IGS of rDNA and ncRNA production from E-pro are, in part, regulated by Top1p.

\author{
Top1Y727Fp restores wild-type levels of H4K16 acetylation at the rDNA locus. \\ Given the different enzymatic activities carried out by Sir2p and Top1p (histone \\ deacetylation versus nicking-religation activity on DNA), the remarkable similarity \\ among reported phenotypes is not easily explained. In order to shed light on these
}


overlapping phenotypes we first asked: is Top1p catalytic activity required to suppress these rDNA phenotypes?

The active site tyrosine of Top1 (Tyr727) acts as a nucleophile to cleave one strand of duplex DNA, and in a transesterification reaction, becomes covalently attached to the 3' phosphoryl end of the nicked DNA strand. Substitution of Phe for Tyr727 (in Top1Y727Fp) results in a catalytically inactive protein that is, nevertheless, still able to bind duplex DNA $(24 ; 25 ; 26)$. In order to evaluate the requirement for Top $1 \mathrm{p}$ catalysis on histone acetylation and ncRNA production, we transformed top1 $\Delta$ cells with a plasmid that constitutively expresses the top $1 Y 727 F$ allele from the TOP1 gene promoter (YCptop1Y727F). A similar plasmid expressing wild-type TOP1 served as control (YCpTOP1). The absence of Top1p catalytic activity in extracts of YCptop1Y727F transformed cells was confirmed in a plasmid DNA relaxation assay using negatively supercoiled plasmid DNA (see Fig. 1S).

The camptothecin (CPT) class of chemotherapeutics specifically targets Top1p, by reversibly stabilizing the covalent enzyme-DNA intermediate formed during the Top1p catalytic cycle $(27 ; 28 ; 29)$. Thus, CPT treatment increases the stability of covalent Top1p-DNA complexes in cells expressing wild-type Top1p, but has no impact on cells expressing the catalytically inactive Top1Y727Fp (18). As shown in Supplemental Fig. 1S, a strong Top1p cleavage site induced by CPT treatment (30) of cells expressing Top1p was not evident in cells expressing the catalytically inactive, and therefore CPT resistant, Top1Y727Fp.

To determine if Top1p catalytic activity was also required to suppress H4K16 acetylation to the levels observed in wild-type cells, top $1 \Delta$ cells transformed with plasmids YCpTOP1 or YCptop1 Y727F were grown in selective media. Wild-type TOP1 
and top1 $1 \Delta$ strains transformed with the empty vector (YCp50) were also analyzed as controls. ChIP analyses were then performed as in Fig. 2A. Fig. 3A depicts the levels of H4K16 acetylation detected in the 5 specified regions described in Fig.1, relative to that observed in wild-type TOP1 cells (dotted line). In contrast to the decrease in H4K16 acetylation detected in the absence of Top1p, H4K16 acetylation was restored to wildtype levels by expression of Top1Y727Fp, across the IGS regions analyzed. Thus, it appears that Top $1 p$ plays a structural, rather than a catalytic role in the maintenance of histone H4K16 acetylation of the IGS of rDNA.

\title{
Top1Y727Fp restores IGS1R ncRNA silencing.
}

As transcription of ncRNAs from E-pro versus C-pro was selectively de-repressed in top1 $\Delta$ cells (20), we next asked if the extent of IGS1R silencing was also restored by Top1Y727F expression. As shown in Fig. 3B, the amount of IGS1R ncRNA expressed (relative to UBC6 and normalized to wild-type TOP1 cells) was equivalent in top1 $\triangle$ transformed with TOP1 and top 1 Y $727 F$ expression vectors. Thus, as with H4K16 deacetylation, these results provide the unexpected observation that DNA topoisomerase I catalytic activity is dispensable for ncRNA silencing.

\author{
Human TOP1 does not restore H4K16 acetylation or ncRNA silencing at the rDNA \\ locus. \\ In eukaryotes, the monomeric nuclear DNA topoisomerase I is highly conserved in \\ terms of enzyme function and architecture. The human nuclear enzyme (hTop1) shares \\ 42\% identity with yeast Top1p (31) (protein alignment in Supplemental Fig. 5S). The \\ conserved core domains of yeast and human Top1p form a protein clamp that
}


completely circumscribes duplex DNA, while a coiled-coil linker domain of variable length connects the core domain with the conserved C-terminal domain, which contains the active site Tyr residue that cleaves the DNA (32; 33; 34; 35). These conserved core and C-terminal domains are $~ 53$ and $62 \%$ identical in amino acid sequence, respectively. Thus, we asked if a structurally related DNA topoisomerase I, human Top1 (encoded by hTOP1 cDNA), could also restore wild-type H4K16 acetylation and ncRNA silencing yeast top $1 \Delta$ cells.

To determine if hTOP1 was able to complement the rDNA phenotypes attendant with TOP1 deletion, GAL1-promoted human TOP1 cDNA constructs (YCpGAL1hTOP1) (34) or yeast TOP1 vector (YCpGAL1-yTOP1) (34), were introduced into top1 cells. The YCp GAL1 empty vector, transformed into wild-type TOP1 or top1 $\triangle$ strains, served as controls. Cells were grown in selective medium containing glucose as carbon source, and TOP1 expression was induced by shifting the cells into galactose containing medium for 6 hours. The activity of the human and yeast enzymes was assessed by the formation of CPT-induced covalent Top1p-DNA complexes in the rDNA in yeast cells, and in plasmid DNA relaxation assays with crude cell extracts (see Supplemental Fig. 2S). We then measured the extent of H4K16 acetylation by ChIP, as described in Figs 2 and 3. As shown in Fig. 4A, the catalytically active human enzyme could not suppress the levels of H4K16 acetylation to those observed in wild-type cells (dotted line) or in top1 $1 \Delta$ cells expressing plasmid-born yeast Top1p (Fig. 4A grey bars). Human Top1p was also unable to restore silencing of transcription at the E-pro (Fig. 4B). RT-PCR quantitation of IGS1R ncRNA production, normalized to UBC6 in wild-type TOP1 cells (dotted line), revealed that ncRNA transcription was unchanged in $\mathrm{h} T O P 1$ 
expressing cells when compared to top $1 \Delta$ cells that contain empty vector, while silencing was restored by the yeast TOP1 gene.

Altogether, the data in Fig. 4 demonstrate that hTOP1 failed to complement the two molecular phenotypes of top1 $\Delta$ cells (H4K16 acetylation and ncRNA derepression). These findings suggest that DNA topoisomerase I catalytic activity is dispensable for ncRNA silencing and histone acetylation control at the rDNA locus, and implicate differences in the protein sequence of these homologous enzymes in the regulation of rDNA phenotypes.

\section{The N-terminal domain of DNA topoisomerase I impacts rDNA phenotypes.}

Our data suggest that distinct features or residues of Top1p, rather than enzyme catalysis, are an important determinant of histone acetylation and silencing at the rDNA locus, as the catalytically inactive yeast Top $1 Y 727 \mathrm{Fp}$ complements the top1 $\Delta$-induced rDNA phenotypes, while an active heterologous hTop1 enzyme does not. As shown in Supplemental Fig. 5S, the N-terminal domains of yeast and human DNA topoisomerase I are poorly conserved, both in terms of size and primary amino acid sequence. No structural information is available for this domain, and it is dispensable for yeast and human Top1p catalysis (36). Nevertheless, the $\mathrm{N}$-terminal domain has been shown to mediate Top1p-protein interactions (29). Thus we asked: is the yeast $\mathrm{N}$-terminal domain required for complementation of top $1 \Delta$ phenotypes?

To address this question, we used a chimeric construct (Sc210hTOP1) that encodes a fusion protein comprising amino acids 1 to 138 from $S$. cerevisiae Top $1 p$ and residues 210 to 765 from the human enzyme, fused at a conserved junction between the $\mathrm{N}$-terminal and core domains of these enzymes. We recently reported that 
swapping of the N-terminal domains of yeast and human Top1p did not impair the specific catalytic activity or CPT sensitivity of the chimeric enzymes (22). A plasmid that expresses the chimeric gene from the galactose-inducible GAL1 promoter (YCpGAL1Sc210hTOP1) was used to transform top1 $\Delta$ cells. Following induction with galactose, the activity of the chimeric enzyme was assessed by the formation of CPT-induced Top1p-DNA covalent complexes at the rDNA locus in vivo and in a plasmid DNA relaxation assays using crude cell extracts (see Supplementary Fig. 3S). These cells were then used in ChIP and RT-PCR analyses, as in Fig. 4. As shown in Fig. 5A, acetylation of H4K16 residues within the IGS was restored to wild-type levels (dotted

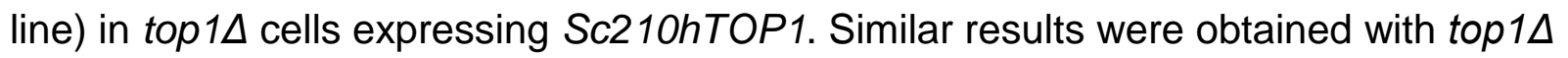
cells transformed with YCpGAL1-yTOP1. The Sc210hTOP1 chimera was also able to restore rDNA silencing, as evidenced by the suppression of IGS1R ncRNA expression (Fig.5B). These results demonstrate that yeast Top1p N-terminal residues 1-138, within the context of the chimeric protein, provide the necessary protein sequences for complementation of the rDNA phenotypes.

\section{DNA topoisomerase I contributes to Sir2p recruitment at the rDNA locus.}

As the $\mathrm{N}$-terminal 138 residues of yeast Top1p suffice to enable human Top1p to complement the rDNA defects in top1 $\Delta$ cells (Fig. 5), we hypothesized that this domain of Top1p acts in concert with other protein partner(s). Moreover, since top1 $\Delta$ and sir2 $\Delta$ mutants exhibit a common loss of gene silencing and histone hyperacetylation at the same locus, we posited that Top $1 p$ recruits Sir2p to the rDNA. To begin addressing this model, ChIP experiments were performed with Sir2p antibodies to analyze regions where Sir2p has previously been reported to bind (35S RNA promoter and RFB, regions 
1 and 5 in Fig. 1) (37). As seen in Fig. 6, Sir2p was enriched at these regions of the rDNA repeats in wild-type TOP1 cells: a two-fold decrease in Sir2p bound to both the 35S RNA promoter and RFB was observed in top1 $1 \Delta$ cells compared to an isogenic wildtype strain (black bars compared to the dotted line in Fig. 6). However, the effect of top1 $1 \Delta$ on Sir2p binding to chromatin appears to be selective for the rDNA locus, as no decrease was evident at other Sir2p binding sites - telomeric sequences of chromosome IV and the HM-L locus (see Supplementary Fig. 4S). However, while Sir2p dependent-silencing at the HML alpha locus was maintained (not shown), the loss of telomeric region silencing in top $1 \Delta$ cells has previously been reported (38). These distinct effects on Sir2p chromatin binding and transcriptional silencing indicate that a global loss of Sir2p activity is not induced by deletion of TOP1.

In Fig. 6A, wild-type levels of Sir2p in rDNA chromatin were restored in top1 $\Delta$ cells that constitutively express plasmid-encoded yeast Top1p or Top1Y727Fp. Conversely, in top1 $1 \Delta$ cells transformed with YCpGAL1-hTOP1, the amount of Sir2p remained the same as that observed in top $1 \Delta$ cells (Fig. 6B). Thus, in agreement with silencing and acetylation phenotypes assessed in Figs. 2-5, the catalytically inactive Top1Y727Fp sufficed to restore Sir2p binding to rDNA, while the active human enzyme did not. Given that the Sc210hTop1 chimera also complemented top1 $\Delta$-induced defects in rDNA silencing and histone acetylation, we next investigated its ability to restore Sir2p binding at rDNA. Indeed, as shown in Fig. 6C, Sir2p levels were also restored in top $1 \Delta$ cells expressing the yeast/human Top1p chimera. In this context, it is worth noting that it was not possible to investigate a possible role of the $\mathrm{N}$-terminal polypeptide alone in recruiting Sir2p to the rDNA in the nucleolus, since it has been reported in human cells that the $\mathrm{N}$-terminal domain (as a single polypeptide) 
translocates from the nucleolus to the nucleoplasm (39), thus preventing any nucleolar specific interactions.

\section{A defined $\mathrm{N}$-terminal region of yeast DNA topoisomerase I rescues ncRNA silencing. \\ To further characterize the specific $\mathrm{N}$-terminal residues of yeast Top1 required to restore rDNA silencing and histone deacetylation, an additional series of previously described N-terminal yeast-human and human-yeast Top1p chimeras were analyzed:} ScA201hTOP1; Sc192hTOP1; h120ScTOP1; h138ScTOP1 (Fig. 7A) (22). YCpGAL1 plasmids containing the chimera gene constructs were transformed into top $1 \Delta$ cells, and IGS1R ncRNA expression was evaluated by RT-PCR. As shown in Fig. 7B, expression of only one additional chimera, Sc $201 h T O P 1$, was able to restore ncRNA silencing. Thus, of the five chimeras examined, only those comprising yeast residues around amino acids 115-125 (in Sc210h and Sc $\Delta 201 \mathrm{~h}$, but not Sc192h) were active in complementing top1 $1 \Delta$-mediated rDNA dysregulation. As shown in Fig. 7A, the common element shared by wild-type yeast Top1p and the two chimeras capable of restoring ncRNA silencing is a continuous stretch of 6 glutamic acidic residues (highlighted yellow). In the case of $\operatorname{Sc} \Delta 201 \mathrm{~h}$, the generation of this six residue acidic patch was a fortuitous consequence of a PCR-induced error in the generation of the chimera (22).

\section{DNA topoisomerase I and Sir2p physically interact}

Given the genetic interaction of yeast Top1p and Sir2p at the rDNA, we next asked if the observed phenotypes result from the physical interaction of these proteins. Protein extracts of isogenic wild-type $\operatorname{SIR} 2$, top $1 \Delta$ or sir2 2 ,top $1 \Delta$ yeast cells expressing the 
indicated yeast, human and chimeric Top1 proteins were subjected to immunoprecipitation (IP) with a bead bound antibody that recognizes the $\mathrm{N}$-terminal FLAG epitope engineered into each TOP1 construct. The bead bound proteins were then immunoblotted with anti-FLAG or anti-Sir2 antibodies to assess coimmunoprecipitation of Sir2p with Top1p. A yeast Topo70 construct was also included in this analysis, as this ScTopo70p lacks the first 102 residues of yeast Top1p, yet still contains the stretch of acidic residues found in the Sc $\Delta 201 \mathrm{~h}$ and Sc210h chimeras (see

Fig. 7A). As seen in Fig. 8, Sir2p was detected following immunoprecipitation in the yeast Top1p, ScTopo70p and chimera samples, with the strongest signal detected with ScTopo70p expressing cells. Conversely, in the IP performed with extracts from cells expressing hTop1p, Sir2p was not detected. Thus, Sir2p appeared to physically interact with all proteins that contain yeast Top $1 p$ residues derived from sequences that span the chimera junctions; yet, Sir2p did not interact with human Top1p. In this regard it is worth noting that all of the Top1 proteins examined, with the exception of human Top1p, contain longer stretches of acidic residues or more repeats of shorter patches of acidic residues than those contained in the human enzyme (Fig. 7A). These elements may suffice to mediate Sir2p binding to Top1p; however, the functional consequence of this interaction on restoring ncRNA silencing is only evident with yeast Top $1 p$, and the Sc $\Delta 201 \mathrm{~h}$ and Sc210h chimeras, which uniquely share a longer acidic patch (EEEEEE).

\section{DISCUSSION}

RNA polymerase II transcriptional silencing at the rDNA locus was first described in $1997(6 ; 7)$, and different gene products have been reported to contribute to this phenomenon (40). The SIR2 gene product appears to comprise the main regulator of 
silencing within this locus, and the histone deacetylase activity of Sir2p is crucial for this function (41). The TOP1 gene has also been implicated in rDNA silencing $(6 ; 7)$. However, the molecular basis of Top1p-mediated regulation of rDNA silencing was an enigma, as the DNA nicking-religation activity of DNA topoisomerase I did not readily suggest involvement in this process.

In order to understand the role of Top1p in RNA polymerase II silencing in rDNA repeats, we first discerned that the enzymatic activity of Top1p was dispensable for rDNA silencing. However, the physical presence of the Top1 protein was essential. In particular, the top $1 \Delta$ mutant defects in ncRNA production and histone acetylation were complemented by plasmid encoded wild-type yeast TOP1, as well as the catalytically inactive yeast top $1 Y 727 F$ mutant. The characteristic hyper-production of ncRNAs and histone hyperacetylation at the IGS region, evident in top $1 \Delta$ cells, were suppressed in both cases, demonstrating that presence of Top1 protein alone sufficed to regulate these two phenotypes. Since Top1Y727Fp binds DNA with the same affinity as wildtype yeast Top1p (26; 42), these findings implicate specific protein interactions with Top1p in Pol II silencing at the rDNA locus.

We further demonstrated that human Top1p, which shares a common reaction mechanism, enzyme architecture, and $42 \%$ identity with the yeast enzyme (31), was unable to restore rDNA silencing or suppress $\mathrm{H} 4 \mathrm{~K} 16$ acetylation in yeast top $1 \Delta$ cells. These findings suggest that specific sequences and/or structural elements unique to yeast Top $1 p$ are required to restore the rDNA defects induced by deletion of the TOP1 gene. A structural role for human Top1 in Pol II transcriptional regulation has also been reported: the catalytic activity of the human enzyme was dispensable for Top1 function in the repression and activation of gene transcription in mammalian cells, yet the yeast 
Top1 protein was not able to complement the loss of human Top1 $(43 ; 44)$. Previous reports further suggest that the DNA topoisomerase I N-terminal domain mediates interactions with different protein partners $(36 ; 45)$. Indeed, using a series of yeast/human chimeras involving reciprocal swaps of the poorly conserved $\mathrm{N}$-terminal domains, our findings demonstrate a pivotal role for select yeast DNA topoisomerase I $\mathrm{N}$-terminal residues in maintaining the silencing and the epigenetic status of the rDNA. Together these data illustrate that: i) Top1p contributes to Sir2p recruitment to rDNA chromatin, but not other loci; ii) the recruitment of Sir2p to the rDNA does not depend on Top1p catalytic activity; and iii) specific N-terminal residues of the yeast enzyme are required for Sir2p recruitment.

The latter point in particular is intriguing and worthy of further consideration. With Sc210hTop1, which comprises the first 138 residues of yeast Top1 fused to amino acid residues 210-765 of human Top1, the catalytic activity and camptothecin sensitivity of the fusion protein mirrored that of wild-type human Top1 (22). In the current studies, Sc210hTop1 expressing cells behaved like wild-type yeast TOP1, strains, demonstrating that the regulation of rDNA silencing and IGS histone H4K16 acetylation is mostly due to the $\mathrm{N}$-terminal sequences of yeast Top1, and correlates with the physical recruitment of Sir2p to the rDNA. These results were not observed with human Top1.

Nevertheless, our data further suggest that a physical interaction of Top1p with Sir2p alone does not suffice to complement the rDNA defects of top $1 \Delta$ cells. First, all of the yeast/human Top1 chimeras examined (Sc210hTOP1; ScL201hTOP1; Sc192hTOP1; h120ScTOP1; and h138ScTOP1), as well as ScTopo70 (which lacks the first 102 residues of yeast Top1), were capable of associating with Sir2p in 

yeast Top1, ScTopo70, and two chimeras, Sc210hTop1p and Sc $\Delta 201 \mathrm{hTop} 1 \mathrm{p}$ sufficed to restore wild-type levels of ncRNA silencing and IGS H4K16 acetylation. In this context, the deletion of 8 residues (EEEDKKAK) in Sc $\Delta 201 \mathrm{hTop} 1 \mathrm{p}$, an unintended error introduced in the generation of this chimera (22), nevertheless provided a critical insight into the functional elements within the $\mathrm{N}$-terminal domain of yeast Top1p. As shown in Fig. 7A, the unique feature shared by the four constructs capable of restoring rDNA silencing and epigenetic regulation by Sir2p, is an $\mathrm{R}$ residue followed by a stretch of seven acidic residues EEEEEED and KKAK in yeast Top1p, ScTopo70p and Sc210hTop1p, or $\mathrm{R}$ followed by six acidic residues EEEEEE and QKWK in Sc $\Delta 201 \mathrm{hTop} 1$. In contrast, Sc192hTop1p, which did not complement the rDNA of top1 $\Delta$ cells, has $\mathrm{R}$ followed by a slightly shorter stretch of five acidic residues EEEEE and KKKPKK (see Fig. 7A). Unfortunately, the corresponding stretch of residues in human Top1p (EPDNKKKPKK) are not resolved in the crystal structures of human Topo70 (33, 35), and acidic/basic patches may not be expected to have well-defined secondary structure in solution. Nevertheless, these residues would be expected to reside on the surface of the protein. Moreover, if arrayed in an alpha-helix, the shorter stretch of acidic residues in Sc192hTop1p (REEEEEKKK) would produce a shift in registry of charge, such that one helical face would be net-positive. In contrast, with the longer acidic stretches in Sc $\Delta 201 \mathrm{hTop} 1 \mathrm{p}$ (REEEEEEQK) or Top1p, ScTopo70p and Sc210hTop1p (REEEEEEDK), all helical faces would be net-negative. These differences could have profound effects on protein interactions with Top1p. An additional possibility is that the presence of a Pro within the adjoining human stretch of Lys residues in Sc192hTop1p (KKKPKK) may alter the structure of the yeast KKAK 
residues to impair critical protein-protein interactions. Although these considerations await additional structural determinations of yeast and human Top1p N-terminal domains, our findings nevertheless lead to the following implications: a role for the $\mathrm{N}$ terminal 138 residues of yeast Top1p in the physical binding of Sir2p; with residues in 115-125 of yeast Top1 required for the functional interactions necessary for complementation of top1 $1 \Delta$-induced rDNA defects. These data further establish the role of DNA topoisomerase I in the epigenetic regulation of rDNA, independent of enzyme catalysis.

\section{MATERIALS AND METHODS}

Yeast strains used in these studies were two pairs of isogenic strains: W303-1a (MATa, ade2-1, ura3-1, his3-11,15, trp1-1, leu2-3,112, can1-100), wild-type for TOP1 and AMR51 (W303-1a; top1 $\Delta:$ LEU2); and EKY2 (MATa, ura3-52, his3 200 , leu2 $\Delta 1$,

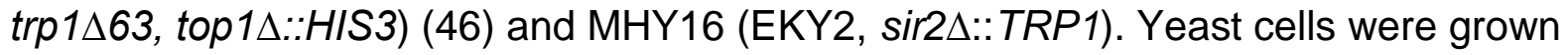
and manipulated according to standard protocols (47). Culture media were YPD (complete media), and YNB without URA/ 2\% glucose or YNB without URA/ 2\% galactose (minimal media).

Plasmids that constitutively express yeast TOP1 or top $1 Y 727 F$ from the yeast TOP1 promoter (YCpTOP1 and YCptop1Y727F, respectively) have been described (18). Galactose-inducible expression of wild-type yeast and human Top1p, yeast Topo70p, and a series of yeast/human and human/yeast $\mathrm{N}$-terminal chimeric enzymes, was achieved with plasmids YCpGAL1yTOP1, YCpGAL1hTOP1, YCpGAL1yTopo70, YCpGAL1Sc192hTOP1, YCpGAL1Sc 2 201hTOP1, YCpGAL1Sc210hTOP1, YCpGAL1h120ScTOP1, and YCpGAL1h138ScTOP1 as described (22). The Sc and $\mathrm{h}$ 
designations indicate the order of yeast and human TOP1 sequences, respectively, while the number refers to the first amino acid residue encoded by the 3' chimera partner. For instance, in Sc210hTOP1, the junction between the N-terminal yeast Top1p sequences and the C-terminal human Top1p sequences begins with human residue 210. The construction of these chimeras was previously reported (22) and a diagram of the sequences flanking the chimeric junctions is provided in Fig. 7A. In all cases, the constructs were engineered with an N-terminal FLAG epitope tag. Oligonucleotides sequences are provided in Supplemental Table T1.

\section{RT-PCR analysis}

RNA from logarithmically growing cultures was isolated as previously described (48). $0.75 \mu \mathrm{g}$ of DNAse I treated RNA (30 min. at $37^{\circ} \mathrm{C}$ ) were subjected to cDNA synthesis with Bioscript ${ }^{\mathrm{TM}}$ Reverse Transcriptase (Bioline reagents Ltd, United Kingdom) for 60 min a $42^{\circ} \mathrm{C}$. UBC6 and IGS1R ncRNA were reverse transcribed using an oligo dT primer. IGS1F ncRNA was reverse transcribed with an IGS1Fr specific primer (Supplemental Table T1). IGS2 ncRNA was reverse transcribed using random hexamers. The reverse transcriptase reaction was stopped by heat inactivation of the enzyme at $85^{\circ} \mathrm{C}$ for $5 \mathrm{~min}$. The resulting cDNA (35 ng) was PCR amplified, using the forward and reverse primers for IGS1R, IGS1F, IGS2, UBC6 (Supplemental Table T1), under the following conditions: denaturation at $95^{\circ} \mathrm{C}$ for $30 \mathrm{sec}$, annealing at $55^{\circ} \mathrm{C}$ for 30 sec and elongation at $68^{\circ} \mathrm{C}$ for $30 \mathrm{sec}$ with 16 cycles for $U B C 6$ and 24 cycles for ncRNAs. Taq polymerase was from Eppendorf (Hamburg, Germany). [ $\left.\alpha{ }^{32} P\right] d A T P$ (Amersham, GE Healthcare) was added to the reaction mixture $(0.04 \mu \mathrm{Ci} / \mu \mathrm{l})$. Template titration for each sample was performed in order to evaluate the linear range of the 
amplification. The amplified fragments were separated on a $6 \%$ acrylamide gel and quantification was performed using ImageJ 1.42q. ncRNA bands intensity was normalized to the UBC6 value. Average (with standard deviations) refers to at least three independent ratios: [mutant or transformed strain ncRNA/ UBC6 RNA]/[wild-type ncRNA/UBC6 RNA]. p-values were obtained using Student's t-test.

\section{Chromatin Immunoprecipitation (ChIP)}

Exponential phase cultures $(200 \mathrm{ml})$ were cross linked with 1\% formaldehyde at room temperature for $15 \mathrm{~min}$ (for acH4K16 or total $\mathrm{H} 4$ immunoprecipitation) or 20 min (for Sir2p immunoprecipitation), and then incubated with $330 \mathrm{mM}$ glycine for $10 \mathrm{~min}$, to quench the formaldehyde reaction. Cells were then processed for ChIP as described (26). Cells were washed with $140 \mathrm{mM} \mathrm{NaCl}, 2.5 \mathrm{mM} \mathrm{KCl}, 8.1 \mathrm{mM} \mathrm{Na}_{2} \mathrm{HPO}_{4}, 1.5 \mathrm{mM} \mathrm{NaCl}$, $1 \mathrm{mM}$ EDTA, 1\%TritonX-100, $0.1 \%$ sodium deoxycholate, $0.1 \%$ SDS, protease inhibitors and lysed with glass beads, by vigorous shaking for $1 \mathrm{~h}$ at $4^{\circ} \mathrm{C}$. Chromatin extracts were recovered and subjected to sonication four times for $20 \mathrm{sec}$ at an amplitude needed to obtain an average DNA fragment size of 500 to $1000 \mathrm{bp}$. Equal amounts of chromatin

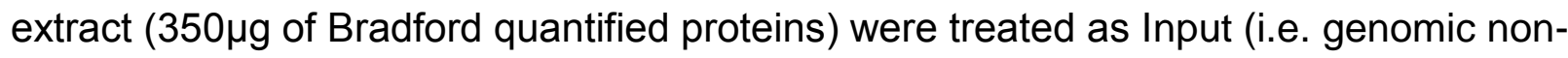
immunoprecipitated DNA) or as IP (Immunoprecipitated DNA). IP samples were incubated at $4^{\circ} \mathrm{C}$ overnight, at the final concentration of $140 \mathrm{mM} \mathrm{NaCl}$, with $100 \mu \mathrm{g}$ of BSA and $2.5 \mu \mathrm{g}$ of antibodies against Sir2p, histone $\mathrm{H} 4$ terminal tail, or acetylated H4K16. Chromatin antibody complexes were isolated with protein A Sepharose beads (Amersham, GE Healthcare) for $1.5 \mathrm{~h}$ at $4^{\circ} \mathrm{C}$. Beads were then washed with lysis buffer containing increasing amounts of $\mathrm{NaCl}$, deoxycholate buffer $(10 \mathrm{mM}$ Tris- $\mathrm{HCl} \mathrm{pH} 8$, $1 \mathrm{mM}$ EDTA). Immunoprecipitated chromatin was then eluted by incubation with $100 \mu \mathrm{l}$ of 
$50 \mathrm{mM}$ Tris- $\mathrm{HCl} \mathrm{pH} 8,10 \mathrm{mM}$ EDTA, $1 \% \mathrm{SDS}$ at $65^{\circ} \mathrm{C}$ for $15 \mathrm{~min}$. All samples (IP and Input) were then incubated at $65^{\circ} \mathrm{C}$ overnight to reverse the crosslinking, treated with $0.5 \mu \mathrm{g} / \mathrm{ml}$ proteinase $\mathrm{K}$ and $0.25 \mu \mathrm{g} / \mathrm{ml}$ RNase A. The recovered DNA was resuspended in $200 \mu$ f for Input samples and in $50 \mu$ for IP samples. $1 \mu$ from Input and $3 \mu$ from IP were used as template for PCR in order to obtain comparable autoradiographic signals. PCR was performed under the following conditions: denaturation at $95^{\circ} \mathrm{C}$ for $30 \mathrm{sec}$, annealing at $55^{\circ} \mathrm{C}$ for $30 \mathrm{sec}$, and elongation at $68^{\circ} \mathrm{C}$ for $30 \mathrm{sec}$, with 17 cycles for IGS rDNA sequences and 27 cycles for ACTIN. $\alpha-{ }^{32} \mathrm{P}$ dATP was added to the reaction mixture $(0.04 \mu \mathrm{Ci} / \mu \mathrm{l})$. Multiplex PCR reactions were performed between: (ENH/ARS/ACT1); (E-pro/C-pro/ACT1); (5S/ACT1). The amplified fragments were separated on a $6 \%$ acrylamide gels and quantification was performed using ImageJ $1.42 \mathrm{q}$.

The fold enrichment values, obtained using antibodies, were calculated as follows: [rDNA(IP)/ACT1(IP)]/ [rDNA(Input)/ACT1(Input)]. Final values relative to acH4K16 were obtained after normalization to total $\mathrm{H} 4$ values. After this calculation, the isogenic wildtype TOP1 strain values were normalized to 1 , obtaining the enrichment amount shown for the different strains or conditions. The graphs show the mean and standard deviation of relative enrichments calculated from three different PCR experiments. p-values were obtained using Student's t-test.

\section{Co-immunoprecipitations}

Galactose-induced cultures of EKY2 (SIR2) or MHY16 (sir2A) cells expressing Nterminal FLAG-tagged Top1p were lysed with prechilled $\left(-20^{\circ} \mathrm{C}\right)$ glass beads in $50 \mathrm{mM}$ 
Tris, pH 7.4, 1 mM EDTA, 1 mM EGTA, $200 \mathrm{mM} \mathrm{KCl}$, and 10\% glycerol, supplemented with complete protease inhibitors (Roche). Triton X-100 was added to a final concentration of $1 \%$. Aliquots were incubated with anti-FLAG agarose beads (Sigma) and rotated end-over-end for 2 hours at $4^{\circ} \mathrm{C}$. The samples were washed five times with $50 \mathrm{mM}$ Tris, $\mathrm{pH} 7.4,200 \mathrm{mM} \mathrm{KCl}$ and protease inhibitors. SDS sample buffer lacking DTT was added to each sample and the proteins were resolved by SDS-PAGE and visualized by immunoblotting with antibodies specific for the FLAG epitope tag onTop1p or Sir2p.

\section{ACKNOWLEDGMENTS}

We thank Dr. Stephen G. Aller for helpful discussions about protein structure.

This work was partially supported by the Istituto Pasteur-Fondazione Cenci Bolognetti Università di Roma La Sapienza, the Epigenomics Flagship Project EpiGen, the Italian Ministry of Education and Research, National Research Council, NIH grant CA58755 and the University of Alabama at Birmingham Comprehensive Cancer Center Core grant P30CA013148.

\section{REFERENCES}

1. Petes, T.D. (1979). Yeast ribosomal DNA genes are located on chromosome XII. Proc. Natl. Acad. Sci. USA. $76: 410-4$.

2. Kobayashi, T., Nomura, M. \& Horiuchi, T. (2001). Identification of DNA cis elements essential for expansion of ribosomal DNA repeats in Saccharomyces cerevisiae. Mol. Cell. Biol. 21: 136-47. 
3. Kobayashi, T. \& Ganley, A.R. (2005). Recombination regulation by transcriptioninduced cohesin dissociation in rDNA repeats. Science. 309: 1581-4.

4. Nomura, M. (2001). Ribosomal RNA genes, RNA polymerases, nucleolar structures, and synthesis of rRNA in the yeast Saccharomyces cerevisiae. Cold Spring Harb Symp Quant Biol. 66: 555-65.

5. Cesarini, E., D'Alfonso, A. \& Camilloni, G. (2012). H4K16 acetylation affects recombination and ncRNA transcription at rDNA in Saccharomyces cerevisiae. Mol. Biol. Cell. 23: 2770-81.

6. Bryk, M., Banerjee, M., Murphy, M., Knudsen, K.E., Garfinkel, D.J.\& Curcio, M.J. (1997). Transcriptional silencing of Ty1 elements in the RDN1 locus of yeast. Genes Dev. 11: 255-69.

7. Smith, J.S. \& Boeke, J.D. (1997). An unusual form of transcriptional silencing in yeast ribosomal DNA. Genes Dev. 11:241-54.

8. Li, C., Mueller, J.E.\& Bryk, M. (2006). Sir2 represses endogenous polymerase II transcription units in the ribosomal DNA nontranscribed spacer. Mol. Biol. Cell. 17: 3848-59. 
09. Gottlieb, S. \& Esposito, R.E. (1989). A new role for a yeast transcriptional silencer gene, SIR2, in regulation of recombination in ribosomal DNA. Cell. 56: 771-6.

10. Kaeberlein, M., McVey, M.\& Guarente, L. (1999). The SIR2/3/4 complex and SIR2 alone promote longevity in Saccharomyces cerevisiae by two different mechanisms. Genes Dev. 13: 2570-80.

11. Robyr, D., Suka, Y., Xenarios, I., Kurdistani, S.K., Wang, A., Suka, N. \& Grunstein, M. (2002). Microarray deacetylation maps determine genome-wide functions for yeast histone deacetylases. Cell. 109: 437-46.

12. Tanny, J.C., Dowd, G.J., Huang, J., Hilz, H. \& Moazed, D. (1999). An enzymatic activity in the yeast Sir2 protein that is essential for gene silencing. Cell. 99: 735-45.

13. Imai, S., Armstrong, C.M., Kaeberlein, M. \& Guarente, L. (2000). Transcriptional silencing and longevity protein Sir2 is an NAD-dependent histone deacetylase. Nature. 403: 795-800.

14. Landry, J., Sutton, A., Tafrov, S.T., Heller, R.C., Stebbins, J., Pillus, L. \& Sternglanz, R. (2000). The silencing protein SIR2 and its homologs are NADdependent protein deacetylases. Proc. Natl. Acad. Sci. USA 97: 5807-11. 
15. Smith, J.S., Brachmann, C.B., Celic, I., Kenna, M.A., Muhammad, S., Starai, V.J. \& Avalos, J.L.,(2000). A philogenetically conserved NAD+ - dependent protein deacetylase activity in the Sir2p protein family. Proc. Natl. Acad. Sci. USA. 97: 6658-63.

16. Christman, M.F., Dietrich, F.S. \& Fink, G.R. (1988). Mitotic recombination in the rDNA of S. cerevisiae is suppressed by the combined action of DNA topoisomerases I and II. Cell. 55: 413-25.

17. Fritze, C.E., Verschueren, K., Strich, R. \& Easton Esposito. R. (1997). Direct evidence for SIR2 modulation of chromatin structure in yeast rDNA. EMBO J. 16: 6495509.

18. Megonigal, M.D., Fertala, J. \& Bjornsti, M-A. (1997). Alterations in the catalytic activity of yeast DNA topoisomerase I result in cell cycle arrest and cell death. J. Biol. Chem. 272: 12801-8.

19. Cioci, F., Vogelauer, M. \& Camilloni, G. (2002). Acetylation and accessibility of rDNA chromatin in Saccharomyces cerevisiae in $\Delta$ top1 and $\Delta$ sir2 mutants. J. Mol. Biol. 322: 41-52.

20. Vasiljeva, L., Kim, M., Mutschler, H., Buratowski, S. \& Meinhart, A. (2008). The Nrd1-Nab3-Sen1 termination complex interacts with the Ser5-phosphorylated RNA polymerase II C-terminal domain. Nat. Struct. Mol. Biol. 15: 795-804. 


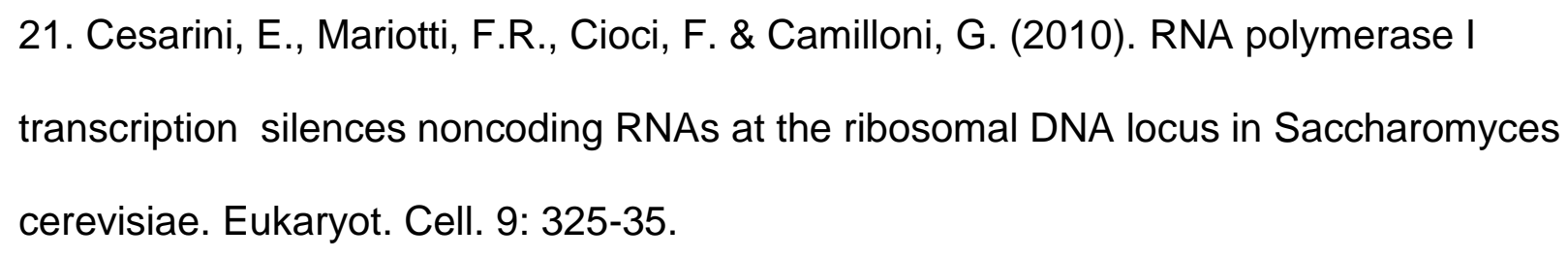

22. Wright, C.M., van der Merwe, M., DeBrot, A.H. \& Bjornsti, M-A. (2015) DNA topoisomerase I domain interactions impact enzyme activity and sensitivity to camptothecin. J. Biol. Chem. 290:12068-78.

23. Teste, M.A., Duquenne, M., François, J.M. \& Parrou, J.L. (2009). Validation of reference genes for quantitative expression analysis by real-time RT-PCR in Saccharomyces cerevisiae. BMC Mol. Biol. 10: 99.

24. Eng, W.K., Pandit, S.D. \& Sternglanz, R. (1989). Mapping of the active site tyrosine of eukaryotic DNA topoisomerase I. J. Biol. Chem. 264: 13373-6.

25. Lynn, R.M., Bjornsti, M-A., Caron, P.R. \& Wang, J.C. (1989) Peptide sequencing and site-directed mutagenesis identify tyrosine-727 as the active site tyrosine of Saccharomyces cerevisiae DNA topoisomerase I. Proc. Natl. Acad. Sci. U S A., 86: 3559-63.

26. Hann, C.L., Carlberg, A.L. \& Bjornsti, M-A. (1998). Intragenic suppressors of mutant DNA topoisomerase l-induced lethality diminish enzyme binding of DNA. J. Biol. Chem. 273:31519-27. 
27. Koster, D.A., Palle, K., Bot, E.S., Bjornsti, M-A. \& Dekker NH. (2007). Antitumour drugs impede DNA uncoiling by topoisomerase I. Nature 448: 213-7.

28. Pommier, Y., Leo, E., Zhang, H. \& Marchand, C. (2010). DNA topoisomerases and their poisoning by anticancer and antibacterial drugs. Chemistry \& Biology 17: 421-33.

29. Chen, S.H., Chan, N.L. \& Hsieh, T.S. (2013). New mechanistic and functional insights into DNA topoisomerases. Annu. Rev. Biochem. 82: 139-70.

30. Vogelauer, M. \& Camilloni, G. (1999). Site-specific in vivo cleavages by DNA topoisomerase I in the regulatory regions of the $35 \mathrm{~S}$ rRNA in Saccharomyces cerevisiae are transcription independent. J. Mol. Biol. 293: 19-28.

31. D'Arpa, P., Machlin, P.S., Ratrie, H. 3rd, Rothfield, N.F., Cleveland, D.W. \& Earnshaw, W.C. (1988). cDNA cloning of human DNA topoisomerase I: catalytic activity of a 67.7-kDa carboxyl-terminal fragment. Proc. Natl. Acad. Sci. USA 85: 2543-7.

32. Stewart, L., Ireton, G.C. \& Champoux, J.J. (1996). The domain organization of human topoisomerase I. J. Biol. Chem. 271: 7602-8.

33. Redinbo, M.R., Stewart, L., Kuhn, P., Champoux, J.J. \& Hol, W.G.J. (1998). Crystal structures of human topoisomerase I in covalent and noncovalent complexes with DNA. Science 279: 1504-13. 
34. Woo, M.H., Losasso, C., Guo, H., Pattarello, L., Benedetti, P. \& Bjornsti. M-A. (2003). Locking the DNA topoisomerase I protein clamp inhibits DNA rotation and induces cell lethality. Proc. Natl. Acad. Sci.USA. 100: 13767-72.

35. Palle, K., Pattarello, L., van der Merwe, M., Losasso, C., Benedetti, P. \& Bjornsti, MA. (2008). Disulfide cross-links reveal conserved features of DNA topoisomerase I architecture and a role for the $\mathrm{N}$ terminus in clamp closure. J. Biol. Chem. 283: 2776-75.

36. Alsner, J., Svejstrup, J.Q., Kjeldsen, E., Sørensen, B.S. \& Westergaard, O. (1992). Identification of an $\mathrm{N}$-terminal domain of eukaryotic DNA topoisomerase I dispensable for catalytic activity but essential for in vivo function. J. Biol. Chem. 267: 12408-11.

37. Huang, J. \& Moazed, D. (2003). Association of the RENT complex with nontranscribed and coding regions of rDNA and a regional requirement for the replication fork block protein Fob1 in rDNA silencing. Genes Dev. 17: 2162-76.

38. Lotito, L., Russo, A., Chillemi, G., Bueno, S., Cavalieri, D. \& Capranico G.(2008). Global transcription regulation by DNA topoisomerase I in exponentially growing Saccharomyces cerevisiae cells: activation of telomere-proximal genes by TOP1 deletion. J. Mol. Biol. 377:311-22.

39. Girstun, A., Kowalska-Loth, B., Czubaty, A., Klocek, M. \& Staroń, K. (2008). Fragment responsible for translocation in the $\mathrm{N}$-terminal domain of human topoisomerase I. Biochem. Biophys. Res. Commun. 366:250-7. 
40. Smith, J.S., Caputo, E. \& Boeke, J.D. (1999). A genetic screen for ribosomal DNA silencing defects identifies multiple DNA replication and chromatin-modulating factors. Mol. Cell. Biol. 19: 3184-97.

41. Blander, G. \& Guarente, L. (2004). The Sir2 family of protein deacetylases. Annu. Rev. Biochem. 73: 417-35.

42. Colley, W.C., van der Merwe, M., Vance, J.R., Burgin, A.B. Jr \& Bjornsti, M-A. (2004). Substitution of conserved residues within the active site alters the cleavage religation equilibrium of DNA topoisomerase I. J. Biol. Chem. 279: 54069-78.

43. Kretzschmar, M., Meisterernst, M. \& Roeder, R.G. (1993). Identification of human DNA topoisomerase I as a cofactor for activator-dependent transcription by RNA polymerase II. Proc. Natl. Acad. Sci. USA. 90: 11508-12.

44. Merino, A., Madden, K.R., Lane, W.S., Champoux, J.J. \& Reinberg, D. (1993). DNA topoisomerase I is involved in both repression and activation of transcription. Nature. 365: 227-32.

45. Edwards, T.K., Saleem, A., Shaman, J.A., Dennis, T., Gerigk, C., Oliveros, E. \& Gartenberg, M.R. (2000). Role for nucleolin/Nsr1 in the cellular localization of topoisomerase I. J. Biol. Chem. 275: 36181-8. 
46. Kauh, E.A. \& Bjornsti, M-A. (1995) SCT1 mutants suppress the camptothecin sensitivity of yeast cells expressing wild-type DNA topoisomerase I. Proc. Natl. Acad. Sci. U S A., 92, 6299-303.

47. Sherman, F., Fink, G.R. \& Lawrence, C. (1983). Methods in Yeast Genetics, Cold Spring Harbor, NY: Cold Spring Harbor Laboratory Press.

48. Verdone, L., Camilloni, G., Mauro, E.D., \& Caserta, M. (1996). Chromatin remodeling during Saccharomyces cerevisiae ADH2 gene activation. Mol. Cell. Biol. 16, 1978-1988. 


\section{Figure Legends}

Figure 1. Schematic map of the ribosomal genes in S. cerevisiae. Horizontal grey arrows represent the 35S RNA pol I and 5 S RNA pol III transcripts. Black arrows represent the ncRNA Pol II transcripts, intergenic spacer IGS1F and IGS1R from E-pro, and IGS2 from C-pro. Filled boxes indicate 35S and 5S coding units. Ellipses refer to positioned nucleosomes. The boxed areas in the lower part of the figure delineate the relative position of DNA sequences used for ChIP experiments, while the arrows indicate the orientation of oligonucleotides used for RT-PCR or primer extension.

\section{Figure 2. top $1 \Delta$ induces increased H4K16 acetylation and the loss of E-pro promoter silencing within the IGS of rDNA.}

A: ChIP analysis of H4K16 acetylation at rDNA. Extracts from crosslinked wild-type and top $1 \Delta$ cells were immunoprecipitated with an anti $-\mathrm{H} 4 \mathrm{~K} 16$ or anti $-\mathrm{H} 4$ antibody. The DNA recovered from the immunoprecipitated chromatin was amplified to analyze 5 rDNA regions specified in Figure 1. H4K16 acetylation data were normalized to total $\mathrm{H} 4$ and then to values obtained with wild-type cells (wild-type $=1$, dotted line). Values with standard deviations from three independent experiments are reported. * $p<0.05$ and ** $\mathrm{p}<0.01$.

B: RT-PCR analysis of ncRNA expression. RNA from wild-type and top1 $\Delta$ strains was analyzed. The levels of ncRNA transcripts were normalized to UBC6 mRNA and then to values obtained from wild-type cells (wild-type $=1$, dotted line). In the histogram, values with standard deviations from three independent experiments are reported. ${ }^{* *} p<0,01$. 
Figure 3. Enzymatic activity of Top1 is dispensable for H4K16 acetylation and ncRNA silencing at IGS.

A: H4K16 acetylation at Enh, E-pro, 5S, ARS, C-pro (regions 1,2,3,4,5 in Fig. 1) were detected by ChIP as in Fig 2. Data from top1 $\Delta$ cells are shown as black bars, from top $1 \Delta$ cells expressing y TOP 1 as grey bars, and from top $1 \Delta$ cells expressing ytop1Y727F (a catalytically inactive mutant of yTOP1) as white bars. Normalized data obtained from wild-type TOP1 cells $=1$ (dotted line). Values with standard deviations from three independent experiments are reported. ${ }^{*} p<0.05{ }^{* *} p<0.01$.

B: IGS1R ncRNA expression assessed by RT-PCR in the same strains as in A, and as described in the legend to Fig. 2. Normalized data from wild-type TOP1 cells $=1$ (dotted line). Values with standard deviations from three independent experiments are reported. ${ }^{*} p<0.05{ }^{* *} p<0.01$

Figure 4. AcH4K16 and silencing of IGS1R are similar in top1D mutants and top1D cells expressing human TOP1.

A: ChIP analysis of H4K16 acetylation, as in Fig. 2 legend. Data from top1 $\Delta$ cells in black bars, from top $1 \triangle$ cells expressing yTOP1 from the GAL1 promoter in grey bars, and from top $1 \triangle$ cells expressing hTOP1 from the GAL1 promoter in white bars. Normalized data from wild-type $T O P 1$ cells $=1$, dotted line. Values with standard deviations from three independent experiments are reported. ${ }^{*} p<0.05{ }^{* *} p<0.01$. B: IGS1R ncRNA levels produced by top $1 \Delta$ yeast cells expressing yeast or human TOP1 (as in A) were assessed by RT-PCR as described in Fig. 2 legend. Normalized wild-type $T O P 1$ strain data $=1$, dotted line, Values with standard deviations from three independent experiments are reported. ${ }^{*} p<0.05{ }^{* *} p<0.01$. 
Figure 5. Sc210htop1 complements top14-induced alterations in H4K16 acetylation and IGS1R ncRNA production.

\begin{abstract}
A: ChIP analysis of acetylated H4K16, as in Fig. 2, was performed in wild-type TOP1 cells (dotted line), top1 $\triangle$ cells (black bars), top1 $\Delta$ cells expressing yTOP1 (grey bars), and top $1 \Delta$ expressing the yeast/human chimera Sc210htop1 (white bars). Values with standard deviations from three independent experiments are reported. ${ }^{*} \mathrm{P}<0.05$, ${ }^{*}$ $\mathrm{P}<0.01$.

B: IGS1R ncRNA transcription detected by RT-PCR in the same strains as in A, as described in Fig. 2 legend. All data were normalized to UBC6 expression, then to data obtained with wild-type TOP1 cells (dotted line); values with standard deviations from three independent experiments are reported. ${ }^{*} p<0.05,{ }^{* *} p<0.01$
\end{abstract}

Figure 6. Sir2p enrichment at rDNA is influenced by the yeast $\mathrm{N}$-terminal domain of Top1p, but not Top1p enzymatic activity. ChIP assays of acetylated H4K16, performed in extracts of top1 $\Delta$ cells (black bars), or top1 $\triangle$ cells expressing plasmid-encoded yTOP1 (dark grey bars), ytop1Y727F (light grey bars), hTOP1 (dot bars) or Sc210htop1 (white bars), subjected to immunoprecipitation with an anti-Sir2p antibody. DNA recovered from chromatin was PCR amplified using oligonucleotides annealing to Enhancer (region 1) or C-pro (region 5) sequences as diagrammed in Fig. 1. All values are normalized to wild-type TOP1 cells (dotted line). Values with standard deviations from three independent experiments are reported. ${ }^{*} p<0.05$ and ${ }^{* *} p<0.01$. 
Figure 7. IGS1R ncRNA transcription in yeast cells expressing chimera TOP1 genes Sc201hTOP1, Sc192hTOP1, h138ScTOP1, h120ScTOP1.

A: Alignment of amino acid residues spanning the chimera junctions of yeast (pink residues) and human (black residues) Top1p, as described in (22). Color coded numbers refer to residue number, while numbered allele designations indicate the first residue of the C-terminal yeast (pink) or human (black) Top1p sequences contained in the chimeric enzymes. In Sc $\Delta 201 \mathrm{~h}$, sequences encoding 8 residues of yeast Top1p, just $\mathrm{N}$-terminal to the chimera junction, were deleted during plasmid construction (indicated by a yellow line). ScTopo70p lacks the first 102 residues and corresponds to human Topo70 constructs that lack the first 174 amino acid residues of human Top1p (35). Stretches of 6 or more acidic residues shared by ScTop1, ScTopo70, Sc $\Delta 201 \mathrm{~h}$ and Sc210h are highlighted in yellow, while shorted patches of Glu residues in the all constructs are in blue.

B: As in Fig. 2 legend, ncRNA expression in top $1 \Delta$ strains expressing the indicated chimera or Top1 protein, was detected by RT-PCR, and normalized to UBC6 and data from wild-type TOP1 cells (dotted line). Values with standard deviations from three independent experiments are reported. ${ }^{*} p<0,05 ;{ }^{* *} p>0,01$.

Figure 8. Yeast Top1p, but not human Top1p, physically interacts with Sir2p. Coimmunoprecipitation analyses were performed with extracts of top1, SIR2 (left panel) or top $1 \Delta$, sir2 $\Delta$ (right panel) cells expressing the indicated yeast, human and chimera Top1 proteins. Lysates were incubated with beads linked to anti-FLAG antibodies. The resulting immunoprecipitates, and input lysate samples were then subjected to 
immunoblot analysis with antibodies specific for the FLAG-tagged Top1 proteins or Sir2p. GAPDH served as loading control for lysates. 

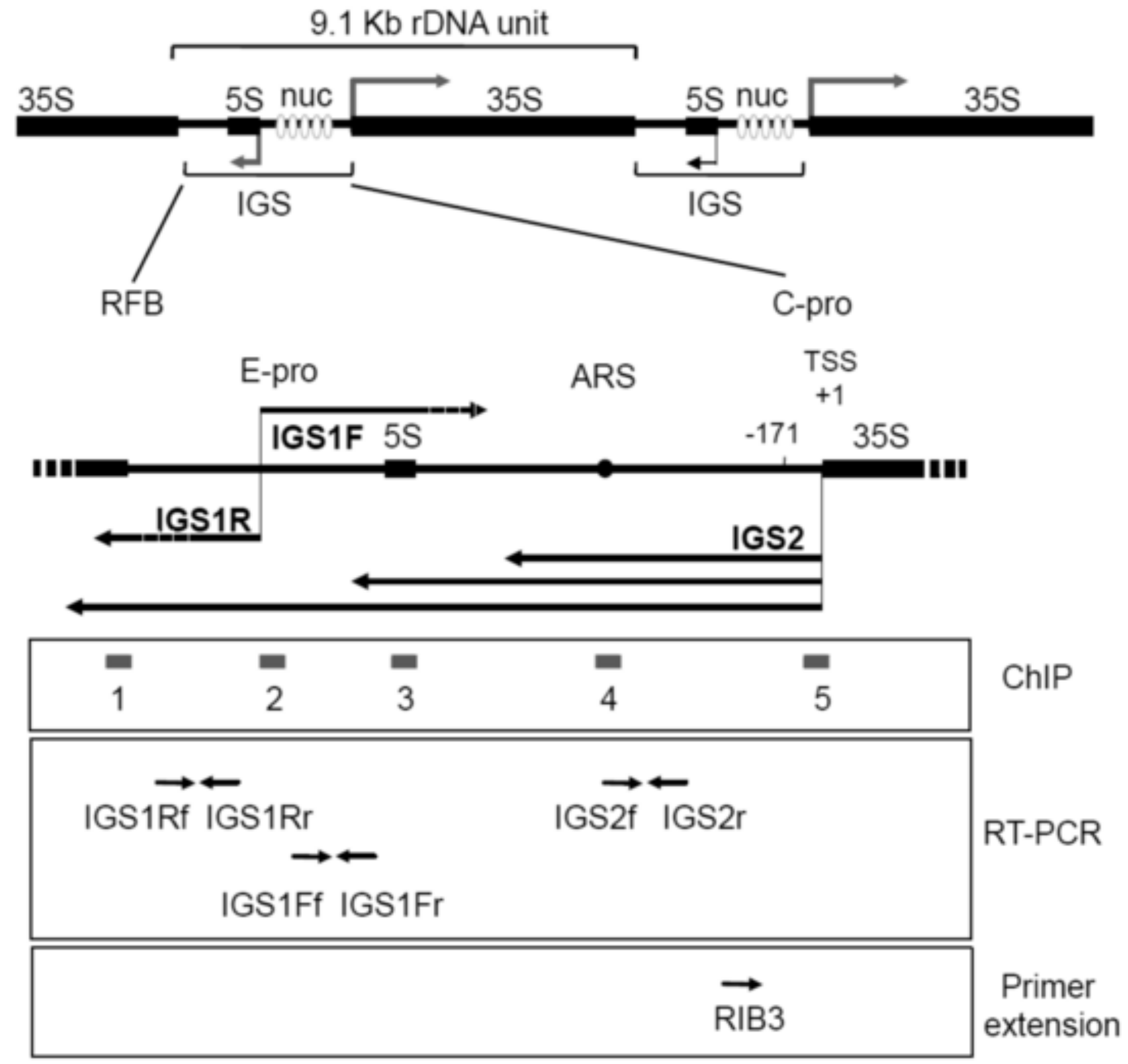
Figure 2

Click here to download high resolution image

A

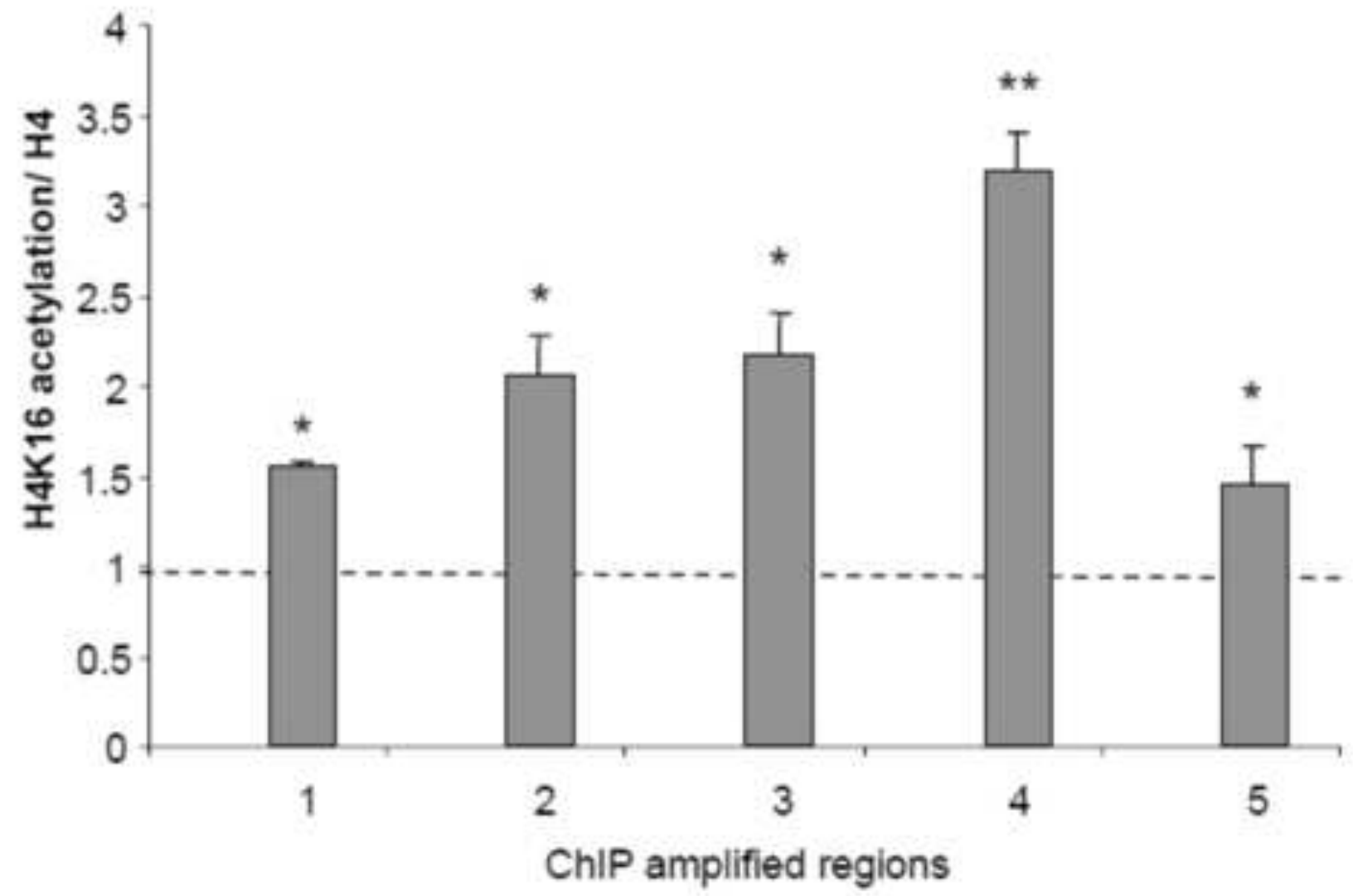

B

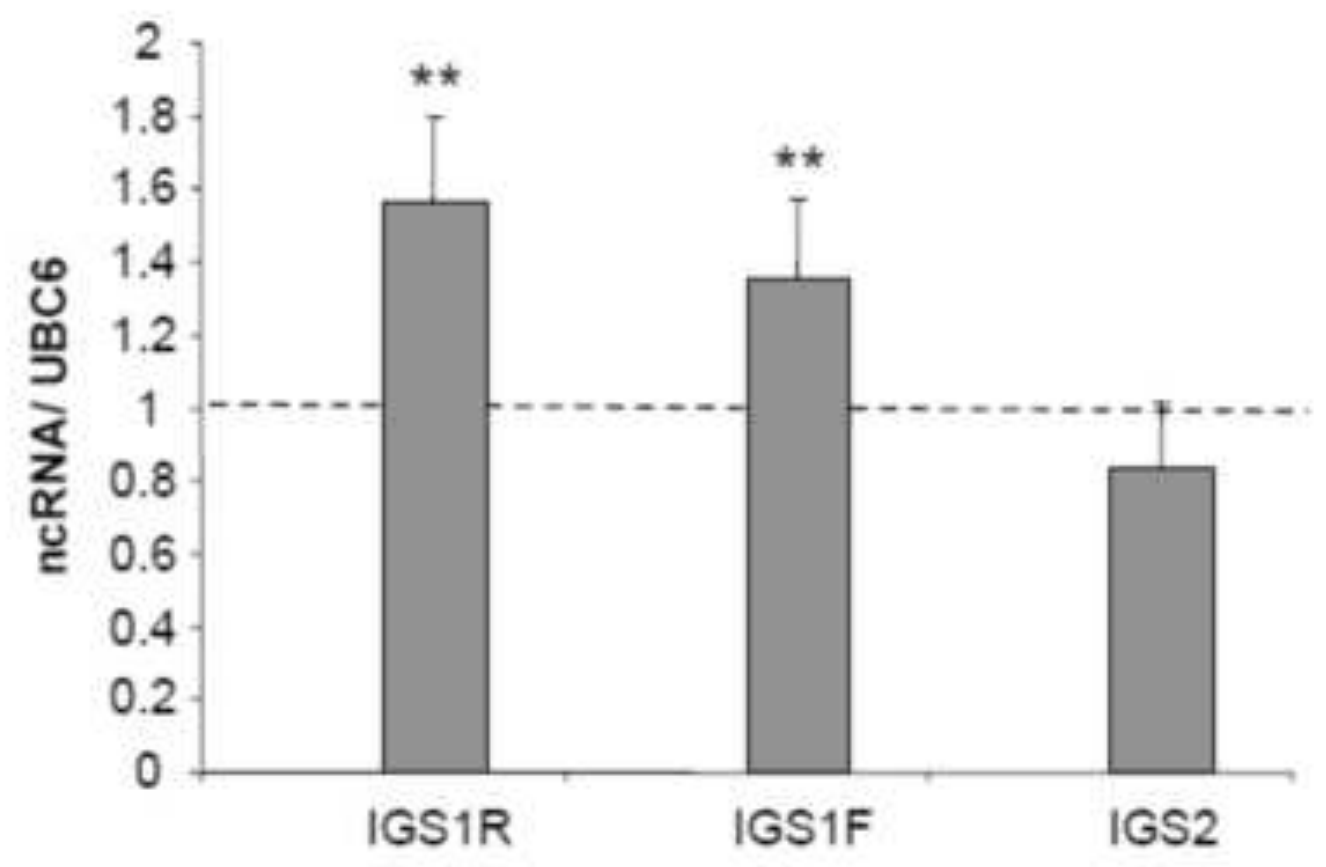


Click here to download high resolution image

A

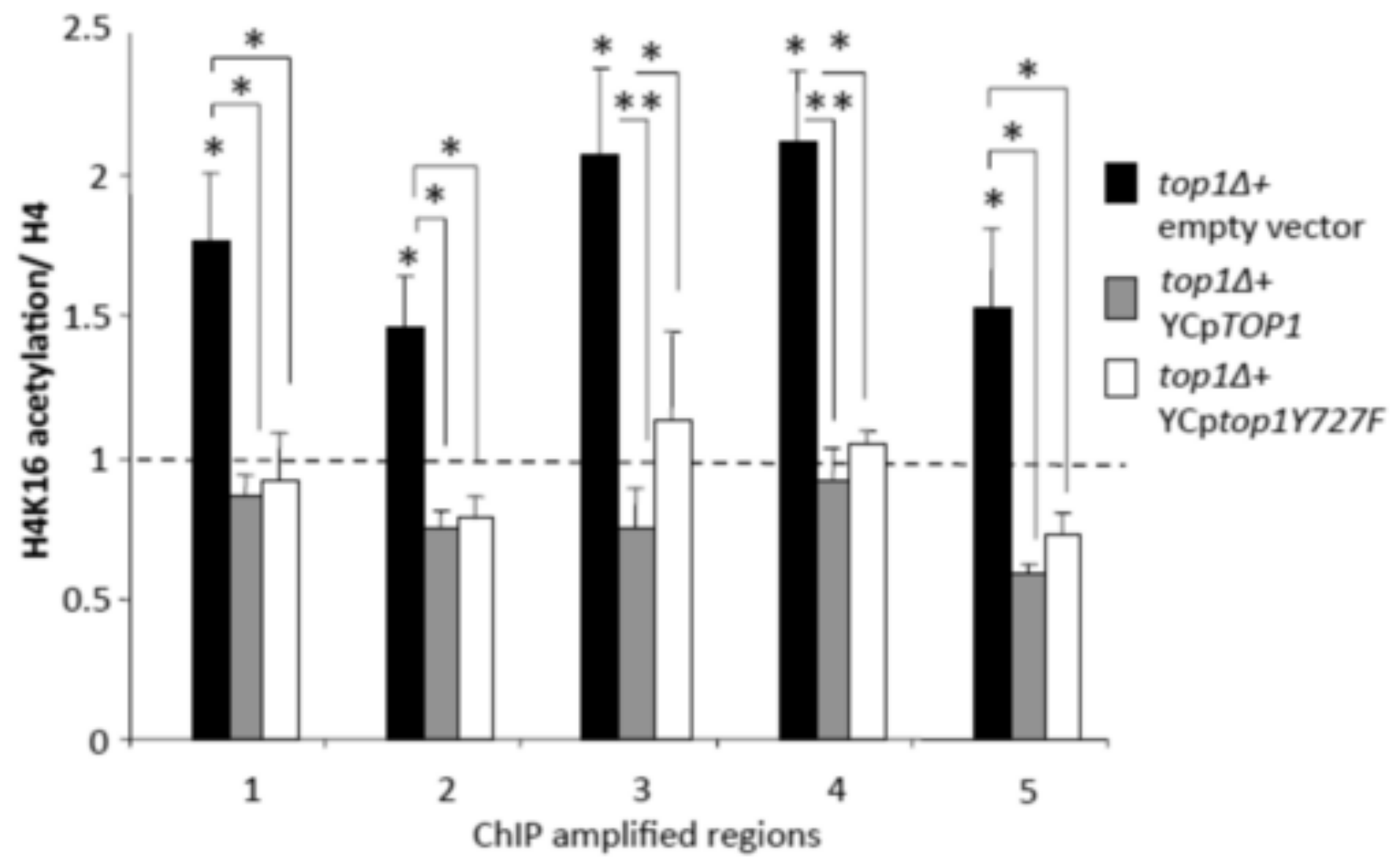

B

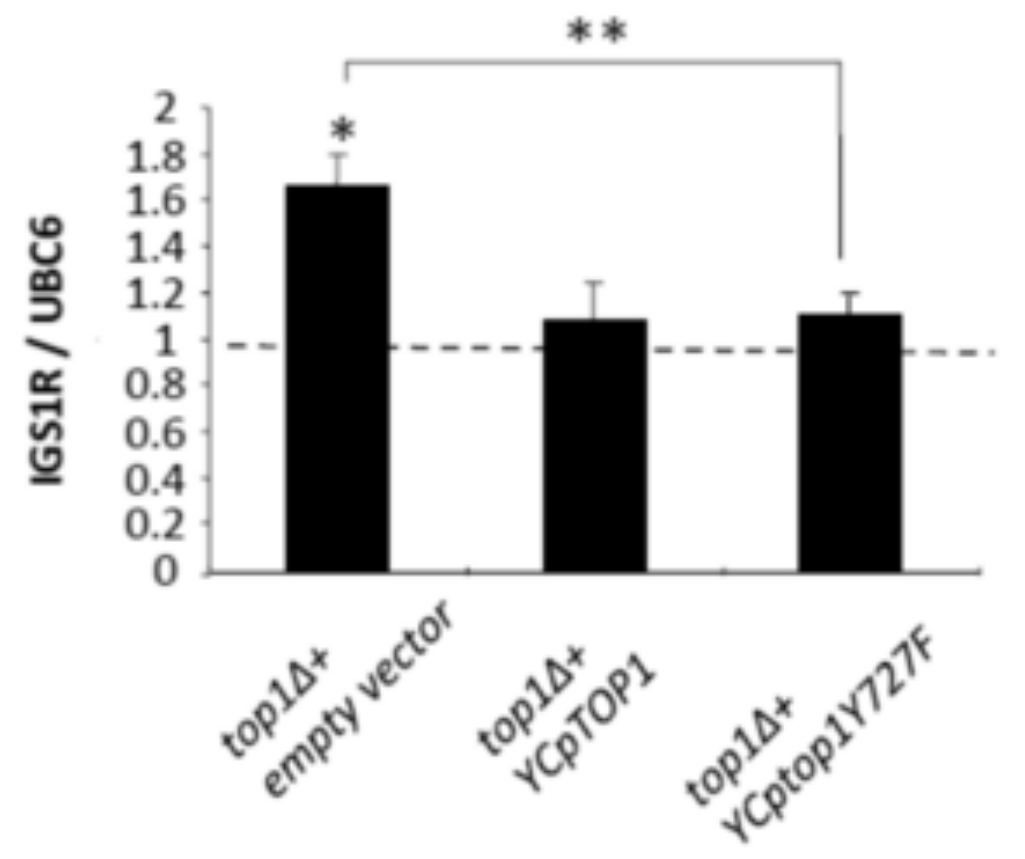




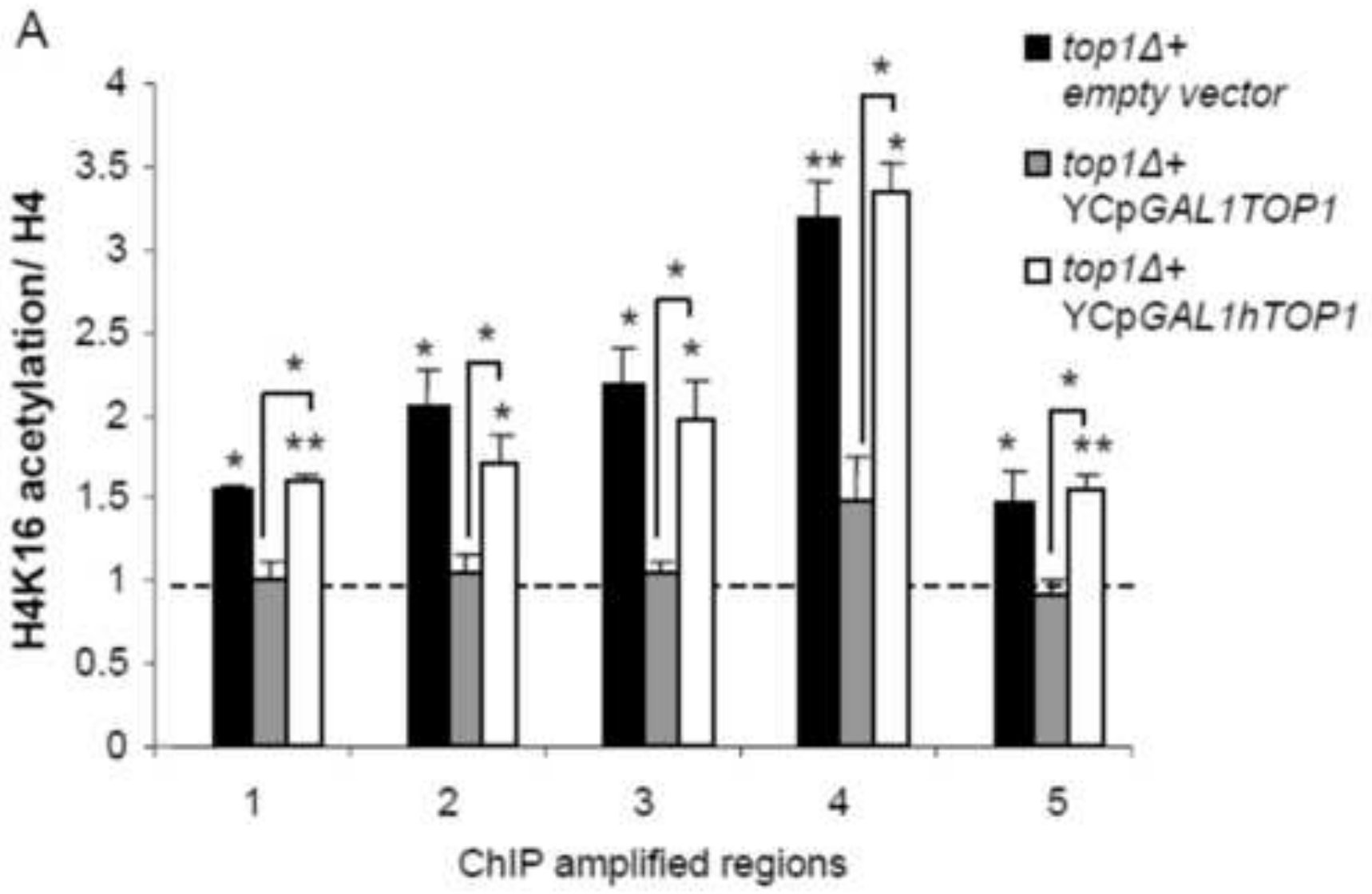

B

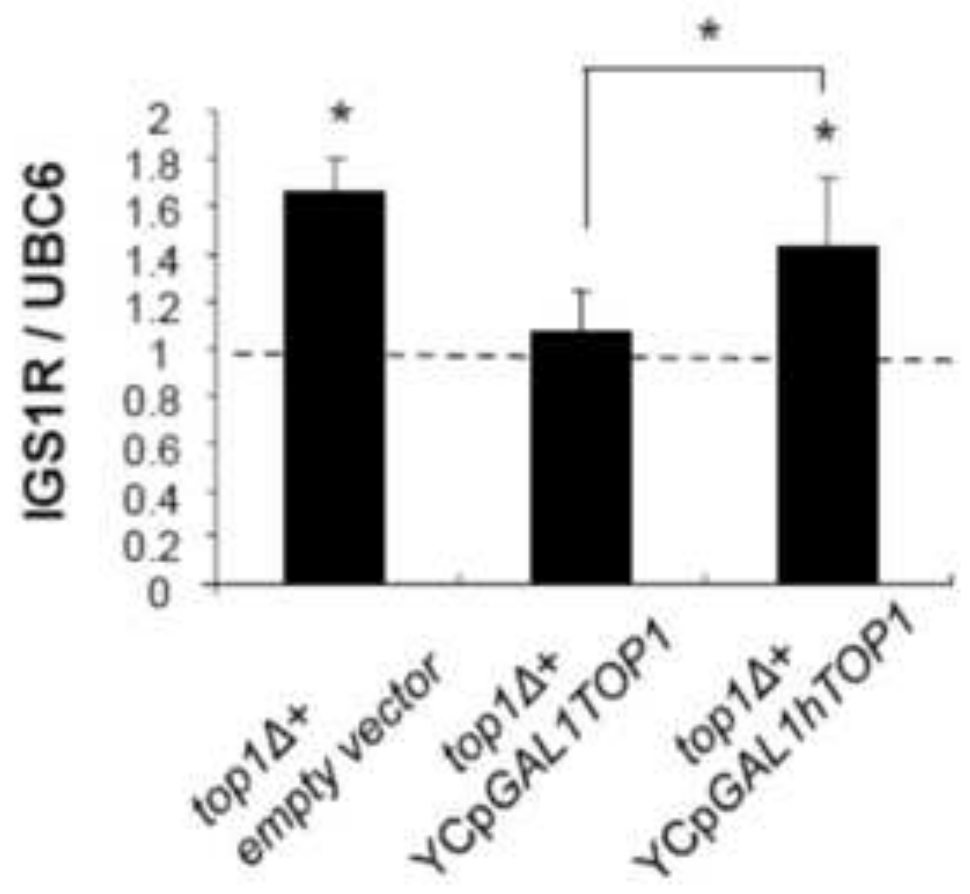


Figure 5
Click here to download high resolution image

A

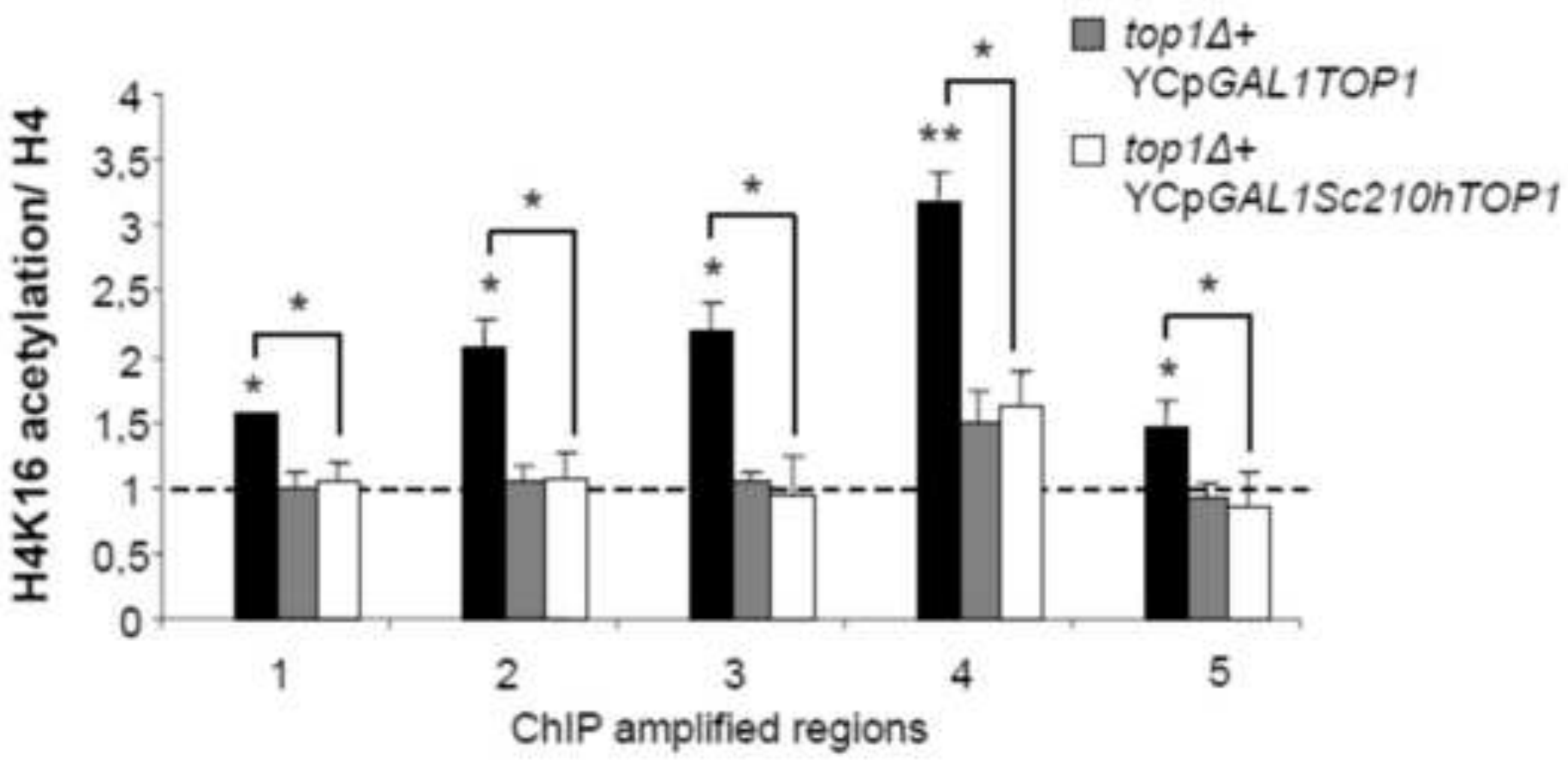

B

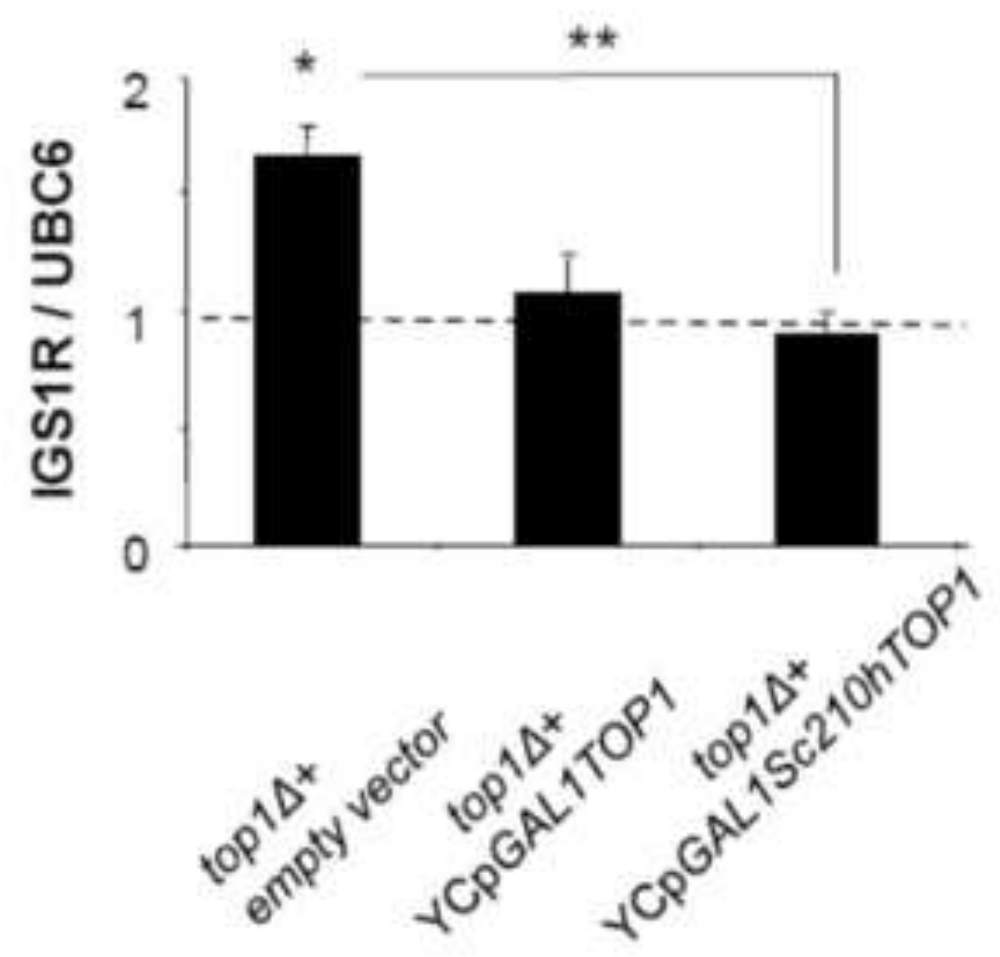


A

B
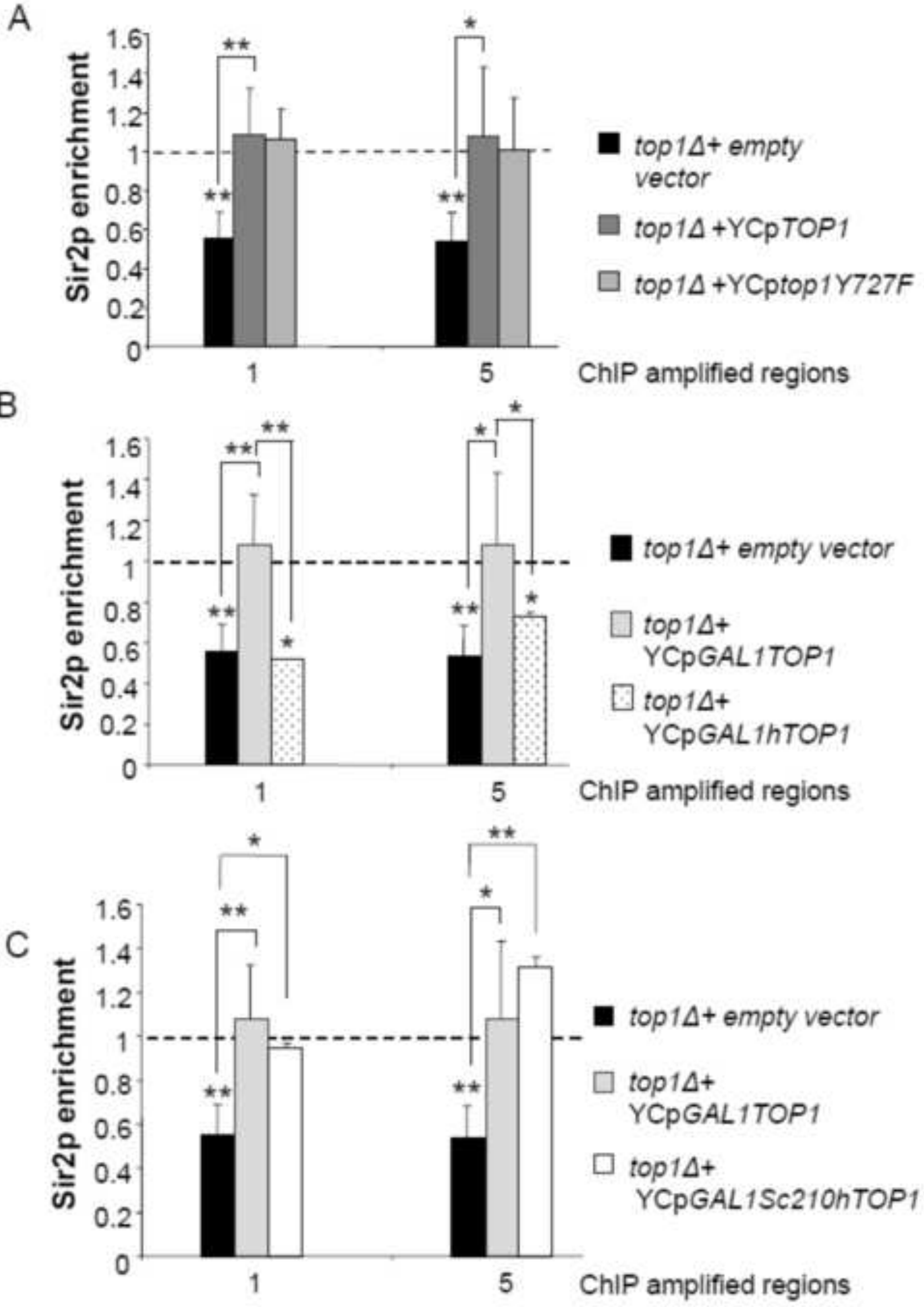
Click here to download high resolution image

A

SoTopl Sclopore

h120Se

h+3ase

solngh

Sctaosh

sezion

hTopt
XTTKXEEQENEKXKREEEEEEOXKAKEEEEEYKWUEKENEDOTIKUNTLKHNGVTFPPPVQ:KKEEQENEKKKKREEEEEEDKKAKEEEEEYKWWEKENEODTIKWVTLKHWGVIFPPPYOKLKKPKNKDKOKKVPEPDNKEDKKAKEEEEEYKWWEXENEDOTIKWNTLKHNGVTFPPPVQKLKKPKNKDKDKKVPEPDNKKKKPKKEEEQXWKWWEEENEDDTIKWVTLKHNOVIPPPPYQXTTKKEEQENEKKKREEEEL KKXPKKEEEQXWKLWEEERYPEGIKWKFLEHKGPVFAPPYE KTTKKEEQENEKXKREEE EEEQKWKWWEERYPEGIKWKFLEHKGPVFAPPYEXTTKKEEQENEKXKREEEEEEDXXAKEEEEEYKWWEKERYPEGIKWKFLEHKGPVFAPPYEXLKKPKNKDKDKKVPEPDNKKKKPKKEEEQKWIKWWEERYPEGIKWKFLEHKGPVFAPPVE-
B

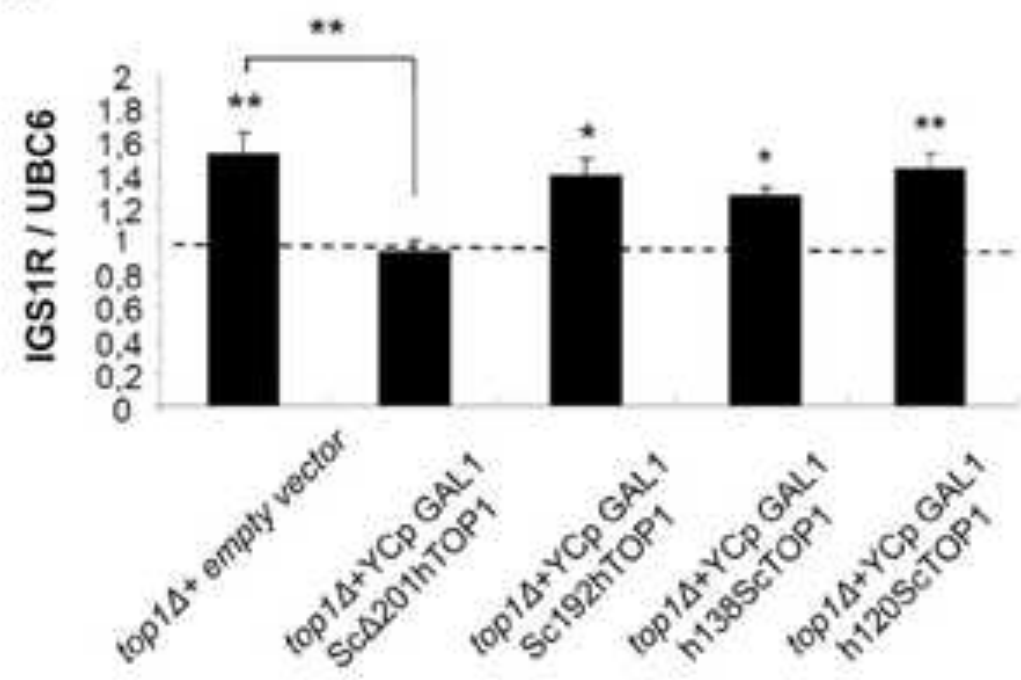


Figure 8

Click here to download high resolution image

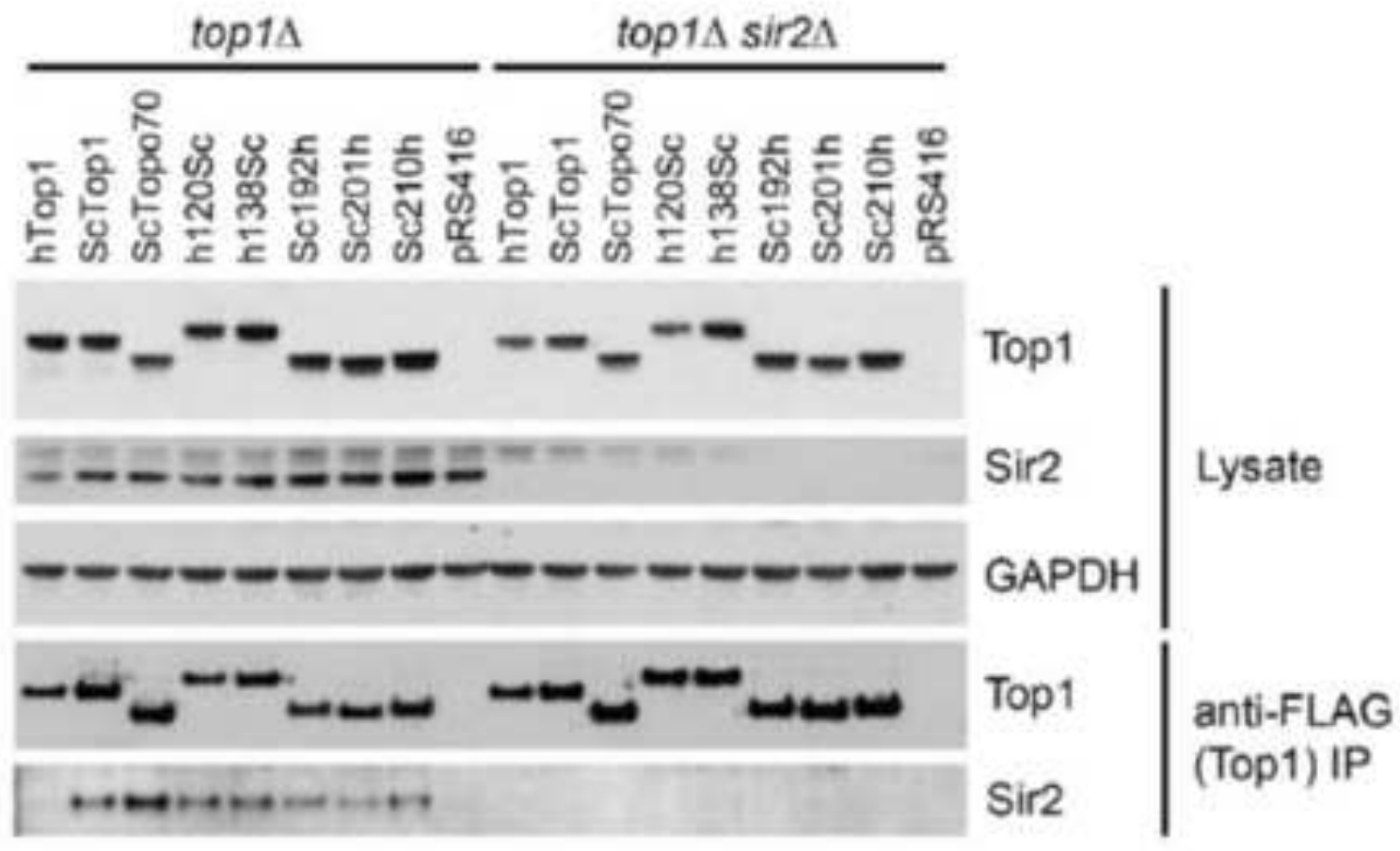


Supplementary Material (Not to be Published)
Click here to download Supplementary Materia

Click here to download Supplementary Material (Not to be Published): JMB_2016 Supplemental Figures.pdf

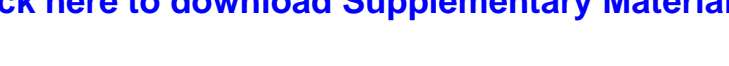


Supplementary Material (Not to be Published)
Click here to download Supplementary Materia

Click here to download Supplementary Material (Not to be Published): JMB_2016 Supplemental Table 1.pdf

C.

(n)

(1)

(1)

(1)

(1)

(1)

.

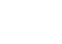

.

.

.

.

.

.

.

.

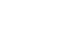

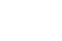

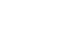

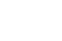

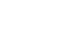

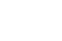

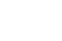

.

.

.

.

.

.

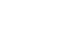

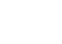

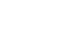

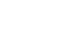

